\title{
On Some Orthogonal Polynomial Integrals
}

\section{By Luigi Gatteschi}

Abstract. The modified moments of the weight functions $w(x)=x^{\rho}(1-x)^{\alpha} \ln (1 / x)$, on $[0,1]$, with respect to the shifted Jacobi polynomials $P_{n}^{*(\alpha, \beta)}(x)=P_{n}^{(\alpha, \beta)}(2 x-1)$, and $w_{p}(x)=x^{\rho} e^{-x}(\ln x)^{p}, p=1,2$, on $[0, \infty)$, with respect to the generalized Laguerre polynomials $L_{n}^{(\alpha)}(x)$, are explicitly evaluated.

1. A Jacobi Polynomial Integral. In a recent paper, Gautschi [3], generalizing a result of Blue [2], has considered and explicitly evaluated the modified moments of the weight function

$$
w(x)=x^{\mu} \ln (1 / x), \quad \mu>-1,
$$

on $[0,1]$, with respect to the shifted Legendre polynomials $P_{n}^{*}(x)=P_{n}(2 x-1)$.

We further generalize these results by considering the weight function

$$
w(x)=x^{\rho}(1-x)^{\alpha} \ln (1 / x), \quad \alpha, \rho>-1,
$$

and evaluating its modified moments on $[0,1]$ with respect to the shifted Jacobi polynomials $P_{n}^{*(\alpha, \beta)}(x)=P_{n}^{(\alpha, \beta)}(2 x-1)$.

It is convenient from now on to replace $\rho$ by $\beta+\mu$; thus, the modified moments we have to examine assume the form

$$
v_{n}^{(\alpha, \beta)}(\mu)=\int_{0}^{1} x^{\beta+\mu}(1-x)^{\alpha} \ln (1 / x) P_{n}^{*(\alpha, \beta)}(x) d x,
$$

$$
\alpha, \beta, \beta+\mu>-1, n=0,1,2, \ldots .
$$

We easily see that

$$
\begin{aligned}
& v_{n}^{(\alpha, \beta)}(\mu)=-2^{-(\alpha+\beta+\mu+1)} \int_{-1}^{1}(1-t)^{\alpha}(1+t)^{\beta+\mu} \ln (1 / 2(1+t)) P_{n}^{(\alpha, \beta)}(t) d t \\
&=-2^{-(\alpha+\beta+\mu+1)}\left\{\int_{-1}^{1}(1-t)^{\alpha}(1+t)^{\beta+\mu} \ln (1+t) P_{n}^{(\alpha, \beta)}(t) d t\right. \\
&\left.-\ln 2 \cdot \int_{-1}^{1}(1-t)^{\alpha}(1+t)^{\beta+\mu} P_{n}^{(\alpha, \beta)}(t) d t\right\},
\end{aligned}
$$

hence, by putting

$$
I_{n}^{(\alpha, \beta)}(\mu)=\int_{-1}^{1}(1-t)^{\alpha}(1+t)^{\beta+\mu} P_{n}^{(\alpha, \beta)}(t) d t,
$$

$$
\alpha, \beta, \beta+\mu>-1, n=0,1,2, \ldots,
$$

Received January 8, 1980.

1980 Mathematics Subject Classification. Primary 33A65. 
we obtain

$$
\nu_{n}^{(\alpha, \beta)}(\mu)=2^{-(\alpha+\beta+\mu+1)}\left\{I_{n}^{(\alpha, \beta)}(\mu) \ln 2-\frac{d}{d \mu} I_{n}^{(\alpha, \beta)}(\mu)\right\} .
$$

The following expression for (1.3),

$$
I_{n}^{(\alpha, \beta)}(\mu)=2^{\alpha+\beta+\mu+1} \frac{\Gamma(\mu+1)}{n ! \Gamma(\mu-n+1)} \frac{\Gamma(\beta+\mu+1) \Gamma(n+\alpha+1)}{\Gamma(n+\alpha+\beta+\mu+2)},
$$

is known ([1], [4, p. 256]). Indeed, (1.5) is easily obtained, multiplying on both sides of Rodrigues' formula,

$$
(1-t)^{\alpha}(1+t)^{\beta} P_{n}^{(\alpha, \beta)}(t)=\frac{(-1)^{n}}{2^{n} n !} \frac{d^{n}}{d t^{n}}\left\{(1-t)^{n+\alpha}(1+t)^{n+\beta}\right\},
$$

by $(1+t)^{\mu}$, integrating from -1 to 1 and carrying out $n$ partial integrations on the right-hand side.

Differentiating (1.5) with respect to $\mu$ gives

$$
\begin{aligned}
\frac{d}{d \mu} I_{n}^{(\alpha, \beta)}(\mu)=I_{n}^{(\alpha, \beta)}(\mu)\{\ln 2 & +\psi(\mu+1)+\psi(\beta+\mu+1) \\
& -\psi(\mu-n+1)-\psi(n+\alpha+\beta+\mu+2)\},
\end{aligned}
$$

where $\psi(x)=\Gamma^{\prime}(x) / \Gamma(x)$ is the logarithmic derivative of the gamma function, and, if $\mu$ coincides with an integer $m<n, m \geqslant 0$, the right-hand member must be replaced by its limit as $\mu \rightarrow m$.

We first consider the case where $\mu \neq 0,1,2, \ldots, n-1$, whenever $n \geqslant 1$. By inserting (1.5) and (1.6) in (1.4), we obtain

$$
\begin{aligned}
\nu_{n}^{(\alpha, \beta)}(\mu)= & \frac{\Gamma(\mu+1) \Gamma(\beta+\mu+1) \Gamma(n+\alpha+1)}{n ! \Gamma(\mu-n+1) \Gamma(n+\alpha+\beta+\mu+2)} \\
& \cdot\{\psi(\mu-n+1)+\psi(n+\alpha+\beta+\mu+2)-\psi(\mu+1)-\psi(\beta+\mu+1)\}
\end{aligned}
$$

with $\alpha, \beta, \beta+\mu>-1, n=0,1,2, \ldots$ and $\mu \neq 0,1,2, \ldots, n-1$ if $n \geqslant 1$.

Taking into account the recurrence relations $\Gamma(x+1)=x \Gamma(x)$ and $\psi(x+1)=$ $\psi(x)+1 / x$, we may derive a useful algorithm for the computation of the modified moments $\nu_{n}^{(\alpha, \beta)}(\mu)$. Indeed, it is easily seen that, if we put

$$
\begin{aligned}
& a_{0}^{(\alpha, \beta)}(\mu)=\frac{\Gamma(\alpha+1) \Gamma(\beta+\mu+1)}{\Gamma(\alpha+\beta+\mu+2)}, \\
& b_{0}^{(\alpha, \beta)}(\mu)=\psi(\alpha+\beta+\mu+2)-\psi(\beta+\mu+1),
\end{aligned}
$$

and we construct the two sequences $\left\{a_{n}^{(\alpha, \beta)}(\mu)\right\}$ and $\left\{b_{n}^{(\alpha, \beta)}(\mu)\right\}$, defined by the recurrence relationships

$$
a_{n}^{(\alpha, \beta)}(\mu)=a_{n-1}^{(\alpha, \beta)}(\mu) \frac{(\alpha+n)(\mu-n+1)}{n(\alpha+\beta+\mu+n+1)},
$$




$$
b_{n}^{(\alpha, \beta)}(\mu)=b_{n-1}^{(\alpha, \beta)}(\mu)+\frac{1}{\alpha+\beta+\mu+1+n}-\frac{1}{\mu+1-n},
$$

we have

$$
\nu_{n}^{(\alpha, \beta)}(\mu)=a_{n}^{(\alpha, \beta)}(\mu) b_{n}^{(\alpha, \beta)}(\mu)
$$

Therefore, this last expression also shows that (1.7) can be written in the following rational form with respect to $n$

$$
\begin{aligned}
& \nu_{n}^{(\alpha, \beta)}(\mu)=\frac{\Gamma(\alpha+1) \Gamma(\beta+\mu+1)}{\Gamma(\alpha+\beta+\mu+2)}\{\psi(\alpha+\beta+\mu+2)-\psi(\beta+\mu+1) \\
& \left.\quad+\sum_{k=1}^{n}\left(\frac{1}{\alpha+\beta+\mu+1+k}-\frac{1}{\mu+1-k}\right)\right\} \\
& \cdot \prod_{k=1}^{n} \frac{(\alpha+k)(\mu+1-k)}{k(\alpha+\beta+\mu+1+k)},
\end{aligned}
$$

where $\alpha, \beta$, and $\mu$ satisfy the above-mentioned conditions.

To examine the remaining case $n \geqslant 1$ and $\mu=m=0,1, \ldots, n-1$, we recall that for any integer $r \geqslant 0$,

$$
\lim _{\epsilon \rightarrow 0} \frac{\psi(-r+\epsilon)}{\Gamma(-r+\epsilon)}=(-1)^{r-1} r !
$$

Then, from (1.7), we obtain

$$
\begin{aligned}
\nu_{n}^{(\alpha, \beta)}(m) & =\lim _{\mu \rightarrow m} v_{n}^{(\alpha, \beta)}(\mu) \\
& =\frac{\Gamma(n+\alpha+1) \Gamma(m+1) \Gamma(\beta+m+1)}{n ! \Gamma(n+\alpha+\beta+m+2)} \lim _{\epsilon \rightarrow 0} \frac{\psi(m+\epsilon-n+1)}{\Gamma(m+\epsilon-n+1)},
\end{aligned}
$$

and finally

$$
\begin{aligned}
& v_{n}^{(\alpha, \beta)}(m)=(-1)^{n-m} \frac{m !(n-m-1) !}{n !} \frac{\Gamma(n+\alpha+1) \Gamma(\beta+m+1)}{\Gamma(n+\alpha+\beta+m+2)}, \\
& \alpha, \beta>-1, m=0,1,2, \ldots, n-1, n \geqslant 1 .
\end{aligned}
$$

This completes the evaluation of the integrals (1.2). Integrals of the form

$$
\int_{0}^{1} x^{\beta+\mu}(1-x)^{\alpha}(\ln (1 / x))^{p} P_{n}^{*(\alpha, \beta)}(x) d x
$$

may be similarly evaluated by repeatedly differentiating (1.7) with respect to $\mu$.

2. Some Examples. The results derived in the previous section show that if one has to evaluate modified moments of a given weight function of type (1.1) for given values of $\rho$ and $\alpha$, then one may choose as polynomial basis the Jacobi polynomials 
$P_{n}^{(\alpha, \beta)}(2 x-1)$, with $\beta$ being a free parameter. For instance, in the case of the weight function

$$
w(x)=x^{\rho} \ln (1 / x), \quad \rho>-1,
$$

we can construct the modified moments associated with the basis $\left\{P_{n}^{(0, \beta)}(2 x-1)\right\}$ instead of the particular one, $\left\{P_{n}^{(0,0)}(2 x-1)\right\}$ considered by Gautschi [3].

It may be of some interest to note that the choice $\rho=\beta$ yields very simple expressions for the corresponding modified moments,

$$
\nu_{n}^{(0, \beta)}(0)=\int_{0}^{1} x^{\beta} \ln (1 / x) P_{n}^{(0, \beta)}(2 x-1) d x= \begin{cases}1 /(\beta+1)^{2}, \quad n=0, \\ \frac{(-1)^{n} \Gamma(\beta+1)(n-1) !}{\Gamma(n+\beta+2)}, & n \geqslant 1 .\end{cases}
$$

Also, in the case of the more general weight functions (1.1), the formulas we obtain are particularly simple when we let $\rho=\beta$,

$$
\begin{aligned}
\int_{0}^{1} x^{\beta}(1-x)^{\alpha} \ln (1 / x) P_{n}^{(\alpha, \beta)}(2 x-1) d x \\
= \begin{cases}\frac{\Gamma(\alpha+1) \Gamma(\beta+1)}{\Gamma(\alpha+\beta+2)}\{\psi(\alpha+\beta+2)-\psi(\beta+1)\}, & n=0, \\
(-1)^{n} \frac{\Gamma(n+\alpha+1) \Gamma(\beta+1)}{n \Gamma(n+\alpha+\beta+2)}, & n \geqslant 1 .\end{cases}
\end{aligned}
$$

An example of (1.1), with $\alpha \neq 0$, could be the weight function

$$
w(x)=x^{\rho}(1-x)^{-1 / 2} \ln (1 / x), \quad \rho>-1,
$$

for which, recalling that $[4$, p. 60$]$

$$
P_{n}^{*(-1 / 2,-1 / 2)}(x)=T_{n}^{*}(x) \prod_{k=1}^{n} \frac{2 k-1}{2 k},
$$

where $T_{n}^{*}(x)=T_{n}(2 x-1)$ is the shifted Chebyshev polynomial of degree $n$. Setting

$$
\tau_{n}(\rho)=\int_{0}^{1} x^{\rho}(1-x)^{-1 / 2} \ln (1 / x) T_{n}^{*}(x) d x, \quad \rho>-1,
$$

and applying (1.9) and (1.8), we have

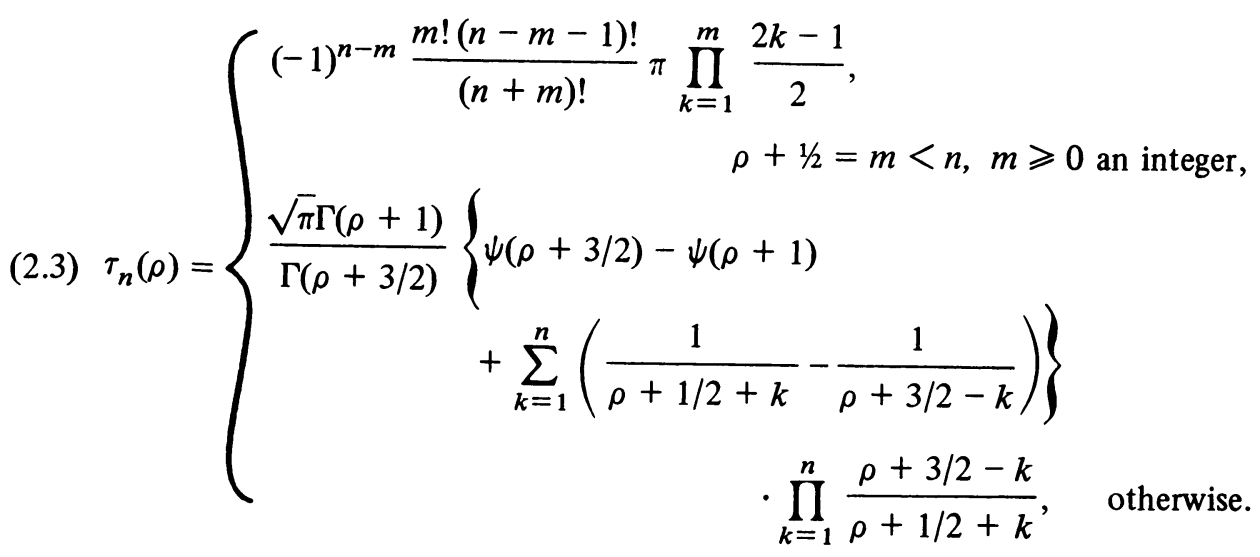


3. Two Laguerre Polynomial Integrals. In this section we consider the problem of evaluating the modified moments of the weight functions

$$
w_{p}(x)=e^{-x} x^{\rho}(\ln x)^{p}, \quad \rho>-1, p=1,2, \ldots,
$$

on $[0, \infty)$, with respect to the generalized Laguerre polynomials $L_{n}^{(\alpha)}(x)$.

We first examine the case $p=1$, which is relative to a weight function of mixed sign. By introducing, for notational convenience, a new parameter $\mu$ such that $\rho=\alpha$ $+\mu$, we refer to the integrals

$$
N_{1, n}^{(\alpha)}(\mu)=\int_{0}^{\infty} e^{-x} x^{\alpha+\mu} \ln x L_{n}^{(\alpha)}(x) d x, \alpha, \alpha+\mu>-1, n=0,1,2, \ldots
$$

for which, if we set

$$
I_{n}^{(\alpha)}(\mu)=\int_{0}^{\infty} e^{-x} x^{\alpha+\mu} L_{n}^{(\alpha)}(x) d x
$$

we have

$$
N_{1, n}^{(\alpha)}(\mu)=\frac{d}{d \mu} I_{n}^{(\alpha)}(\mu)
$$

The evaluation of $I_{n}^{(\alpha)}(\mu)$, hence of $N_{1, n}^{(\alpha)}(\mu)$, can be carried out in a way much similar to that concerning the Jacobi polynomials described in Section 1. To this end, we need to recall the Rodrigues formula for Laguerre polynomials

$$
e^{-x} x^{\alpha} L_{n}^{(\alpha)}(x)=\frac{1}{n !} \frac{d^{n}}{d x^{n}}\left(e^{-x} x^{n+\alpha}\right) .
$$

Indeed, inserting this last formula in (3.3) and integrating by parts $n$ times, we obtain

$$
I_{n}^{(\alpha)}(\mu)=\frac{(-1)^{n}}{n !} \frac{\Gamma(\mu+1) \Gamma(\mu+\alpha+1)}{\Gamma(\mu-n+1)} .
$$

At this point it is not difficult to derive from (3.4) the following two expressions

$$
\begin{aligned}
N_{1, n}^{(\alpha)}(\mu)= & \frac{(-1)^{n}}{n !} \frac{\Gamma(\mu+1) \Gamma(\mu+\alpha+1)}{\Gamma(\mu-n+1)} \\
& \cdot\{\psi(\mu+1)+\psi(\mu+\alpha+1)-\psi(\mu-n+1)\},
\end{aligned}
$$

with $\alpha, \alpha+\mu>-1, n=0,1,2, \ldots$ and $\mu \neq 0,1, \ldots, n-1$ if $n \geqslant 1$, and

$$
N_{1, n}^{(\alpha)}(m)=(-1)^{m-1} \frac{m !(n-m-1) !}{n !} \Gamma(m+\alpha+1),
$$

$$
m=0,1, \ldots, n-1, n \geqslant 1 ;
$$

the second being obtained from the first by taking its limit as $\mu \rightarrow m$. 
Finally, we remark that (3.5) may be put in the following form

$$
N_{1, n}^{(\alpha)}(\mu)=\Gamma(\mu+\alpha+1)\left\{\psi(\mu+\alpha+1)-\sum_{k=1}^{n} \frac{1}{k-\mu-1}\right\} \prod_{k=1}^{n} \frac{k-\mu-1}{k}
$$

The evaluation of the modified moments

$$
N_{p, n}^{(\alpha)}(\mu)=\int_{0}^{\infty} e^{-x} x^{\alpha+\mu}(\ln x)^{p} L_{n}^{(\alpha)}(x) d x, \quad p \geqslant 2
$$

associated to the weight functions (3.1), can be obtained by repeatedly differentiating (3.5) with respect to $\mu$. We shall only examine, with some details, the case $p=2$.

Differentiating (3.5) once, with respect to $\mu$, gives

$$
\begin{aligned}
N_{2, n}^{(\alpha)}(\mu)= & \frac{(-1)^{n}}{n !} \frac{\Gamma(\mu+1) \Gamma(\mu+\alpha+1)}{\Gamma(\mu-n+1)} \\
& \cdot\left\{(\psi(\mu+1)+\psi(\mu+\alpha+1)-\psi(\mu-n+1))^{2}\right. \\
& \left.\quad+\psi^{\prime}(\mu+1)+\psi^{\prime}(\mu+\alpha+1)-\psi^{\prime}(\mu-n+1)\right\},
\end{aligned}
$$

which holds for all $n \geqslant 0$ with $\mu \neq 0,1,2, \ldots, n-1$, when $n \geqslant 1$.

A more convenient form of (3.8), obtained by using the previously recalled properties of the functions $\Gamma(x)$ and $\psi(x)$, together with the recurrence relation $\psi^{\prime}(x+1)=\psi^{\prime}(x)-1 / x^{2}$, is

$$
N_{2, n}^{(\alpha)}(\mu)=\Gamma(\mu+\alpha+1)\left\{\left(\psi(\mu+\alpha+1)-\sum_{k=1}^{n} \frac{1}{k-\mu-1}\right)^{2}\right.
$$

$$
\left.+\psi^{\prime}(\mu+\alpha+1)-\sum_{k=1}^{n} \frac{1}{(k-\mu-1)^{2}}\right\} \prod_{k=1}^{n} \frac{k-\mu-1}{k} .
$$

If $\mu=m=0,1,2, \ldots, n-1, n \geqslant 1$, from (3.8) we have

$$
N_{2, n}^{(\alpha)}(m)=\lim _{\mu \rightarrow m} N_{2, n}^{(\alpha)}(\mu)=\frac{(-1)^{n}}{n !} \Gamma(m+1) \Gamma(m+\alpha+1)\left\{A_{n}(m)-2 B_{n}(m)\right\}
$$

where

$$
\begin{aligned}
& A_{n}(m)=\lim _{\epsilon \rightarrow 0} \frac{\psi^{2}(m+\epsilon-n+1)-\psi^{\prime}(m+\epsilon-n+1)}{\Gamma(m+\epsilon-n+1)} \\
& B_{n}(m)=\lim _{\epsilon \rightarrow 0}\{\psi(m+\epsilon+1)+\psi(m+\epsilon+\alpha+1)\} \frac{\psi(m+\epsilon-n+1)}{\Gamma(m+\epsilon-n+1)}
\end{aligned}
$$

By means of the two series expansions

$$
\begin{array}{ll}
\Gamma(x)=\frac{(-1)^{r}}{r !} \frac{1}{x+r}+\sum_{k=0}^{\infty} a_{k}(x+r)^{k}, & r=0,1,2, \ldots, \\
\psi(x) & =\frac{-1}{x+r}+\psi(1+r)+\sum_{k=0}^{\infty} b_{k}(x+r)^{k},
\end{array}
$$


which are valid for $|x+r|<1$, it is easily seen that

$$
\begin{aligned}
& A_{n}(m)=(-1)^{n-m} 2(n-m-1) ! \psi(n-m), \\
& B_{n}(m)=(-1)^{n-m}(n-m-1) !\{\psi(m+1)+\psi(m+\alpha+1)\} .
\end{aligned}
$$

Hence, substituting these last two expressions into (3.10), we obtain the final result

$$
\begin{aligned}
N_{2, n}^{(\alpha)}(m)= & (-1)^{m} 2 \frac{m !(n-m-1) !}{n !} \Gamma(m+\alpha+1) \\
& \cdot\{\psi(n-m)-\psi(m+1)-\psi(m+\alpha+1)\}, \\
& \quad m=0,1, \ldots, n-1, n \geqslant 1 .
\end{aligned}
$$

4. Some Particular Cases. The results derived in Section 3 assume a very simple form when $\mu=0$, that is in the cases where the weight functions

$$
e^{-x} x^{\alpha} \ln x \quad \text { and } \quad e^{-x} x^{\alpha}(\ln x)^{2}, \quad \alpha>-1,
$$

and the polynomials $L_{n}^{(\alpha)}(x)$ have the same parameter $\alpha$.

For the first weight function, applying (3.7) and (3.6), we find

$$
\int_{0}^{\infty} e^{-x} x^{\alpha} \ln x L_{n}^{(\alpha)}(x) d x= \begin{cases}\Gamma(\alpha+1) \psi(\alpha+1), & n=0, \\ -\Gamma(\alpha+1) / n, & n \geqslant 1,\end{cases}
$$

which may be regarded as a generalization of the well-known integral representation

$$
\gamma=-\int_{0}^{\infty} e^{-x} \ln x d x
$$

of the Euler-Mascheroni constant $\gamma=-\psi(1)=.5772156649 \ldots$

For the second weight function, by using (3.9) and (3.11), we obtain

$$
\int_{0}^{\infty} e^{-x} x^{\alpha}(\ln x)^{2} L_{n}^{(\alpha)}(x) d x= \begin{cases}\Gamma(\alpha+1)\left\{\psi^{2}(\alpha+1)+\psi^{\prime}(\alpha+1)\right\}, & n=0 \\ \frac{2}{n} \Gamma(\alpha+1)\left\{\sum_{k=1}^{n-1} \frac{1}{k}-\psi(\alpha+1)\right\}, & n \geqslant 1\end{cases}
$$

Two other cases of interest, which may be used, for instance, in constructing the modified moments of the weight functions

$$
\exp \left(-x^{2}\right) \ln |x| \text { and } \exp \left(-x^{2}\right)(\ln |x|)^{2}
$$

on $(-\infty, \infty)$, with respect to the Hermite polynomials $H_{n}(x)$, are obtained by means of Szegö's relationships [4, p. 106] between Hermite and Laguerre polynomials,

$$
H_{2 n}(x)=(-1)^{n} 2^{2 n} n ! L_{n}^{(-1 / 2)}\left(x^{2}\right), \quad H_{2 n+1}(x)=(-1)^{n} 2^{2 n+1} n ! x L_{n}^{(1 / 2)}\left(x^{2}\right) .
$$

Indeed, setting $x=t^{2}$ in the integral (3.2), with $\alpha=-1 / 2$ and $\mu=0$, from (3.7) and (3.6) we obtain

$$
4 \int_{0}^{\infty} \exp \left(-t^{2}\right) \ln t L_{n}^{(-1 / 2)}\left(t^{2}\right) d t=\left\{\begin{array}{l}
\sqrt{\pi} \psi(1 / 2)=-\sqrt{\pi}(\gamma+2 \ln 2), \quad n=0, \\
-\sqrt{\pi} / n, \quad n \geqslant 1,
\end{array}\right.
$$


hence, by applying the first relation in (4.3),

$$
\int_{0}^{\infty} \exp \left(-x^{2}\right) \ln x H_{2 n}(x) d x=\left\{\begin{array}{l}
\frac{-\sqrt{\pi}}{4}(\gamma+2 \ln 2), \quad n=0, \\
(-1)^{n-1}(n-1) ! 2^{2(n-1)} \sqrt{\pi}, \quad n \geqslant 1 .
\end{array}\right.
$$

Similarly, if we assume $\alpha=1 / 2$ and $\mu=-1 / 2$ in (3.2), then (3.7), together with the second relation in (4.3), gives

$$
\begin{aligned}
\int_{0}^{\infty} \exp \left(-x^{2}\right) \ln x H_{2 n+1}(x) d x \\
=(-1)^{n-1} 2^{n-1}\left(\gamma+2 \sum_{k=1}^{n} \frac{1}{2 k-1}\right) \prod_{k=1}^{n}(2 k-1), \\
\quad n=0,1,2, \ldots .
\end{aligned}
$$

The integrals involving the weight function $\exp \left(-x^{2}\right)(\ln x)^{2}$ can be dealt with in the same way. Recalling that $\psi^{\prime}(1 / 2)=\pi^{2} / 2$ and $\psi^{\prime}(1)=\pi^{2} / 6$, by use of (3.9) and (3.11), this leads to the following results,

$$
\begin{aligned}
& \int_{0}^{\infty} \exp \left(-x^{2}\right)(\ln x)^{2} H_{2 n}(x) \\
& \quad= \begin{cases}\frac{\sqrt{\pi}}{8}\left\{\psi^{2}(1 / 2)+\psi^{\prime}(1 / 2)\right\}=1.9475221803 \ldots, & n=0 \\
(-1)^{n} 2^{2(n-1)}(n-1) ! \sqrt{\pi}\left(\gamma+2 \ln 2+\sum_{k=1}^{n-1} \frac{1}{k}\right), & n \geqslant 1,\end{cases}
\end{aligned}
$$

and

$$
\begin{array}{r}
\int_{0}^{\infty} \exp \left(-x^{2}\right)(\ln x)^{2} H_{2 n+1}(x) d x \\
=(-1)^{n} 2^{n-2}\left\{\left(\gamma+\sum_{k=1}^{n} \frac{2}{2 k-1}\right)^{2}+\frac{\pi^{2}}{6}-\sum_{k=1}^{n} \frac{4}{(2 k-1)^{2}}\right\} \prod_{k=1}^{n}(2 k-1), \\
n=0,1,2, \ldots
\end{array}
$$

Istituto di Calcoli Numerici

Università di Torino

Via C. Alberto, 10

I-10123 Torino, Italy

1. R. ASKEY \& B. RAZBAN, "An integral for Jacobi polynomials," Simon Stevin, v. 46, $1972 / 1973$, pp. $165-169$.

2. J. L. BLUE, “A Legendre polynomial integral," Math. Comp., v. 33, 1979, pp. 739-741.

3. W. GAUTSCHI, “On the preceding paper 'A Legendre polynomial integral' by J. L. Blue," Math. Comp., v. 33, 1979, pp. 742-743.

4. G. SZEGÖ, Orthogonal Polynomials, 4th ed., Amer. Math. Soc. Colloq. Publ., vol. 23, Amer. Math. Soc., Providence, R. I., 1975. 